\title{
Les facies gypso-salins dans la région d'Oran et leurs conséquences sur les ressources
}

\author{
BELLAREDJ Abderahim El Mehdi \\ Faculté des Sciences de la Terre et de l'univers. Université d'Oran 2 Mohammed Ben Ahmed BP 1015 El \\ mnaouer 31000 \\ Email : bellaredj@yahoo.fr / Téléphone : 00213041556122
}

Original submitted in on $18^{\text {th }}$ April 2016. Published online at www.m.elewa.org on 31st August 2016

http://dx.doi.org/10.4314/jab.v104i1.10

\section{RÉSUMÉ :}

Plus de quarante mille hectares (sols de la plaine de la Mleta et la sebkha d'Oran) et des immenses réserves en eau sont contaminés par le sel.

Objectif : Le présent travail vise à démontrer que la salinisation actuelle de la ressource (sols et eaux) dans la région d'Oran est due principalement aux formations salifères (datant du Trias, Lias, Éocène, Miocène, Pliocène), incorporées aux massifs montagneux (après plissement de l'Atlas Tellien).

Méthodologie et résultats: L'analyse classique, l'approche géochimique et l'étude géomorphologique démontrent que les évaporites sont issues d'un substrat congénitalement salé (salinisation primaire) que les eaux de pluie entraînent en aval (salinisation secondaire des sols et des nappes).

Conclusion et application des résultats : La salinité associée à l'érosion (hydrique et éolienne du fait de l'absence de végétation) au fil du temps, a non seulement affecté les terres et les nappes, mais a contribué à la mise en place d'un synclinal ou sebkha en raison de la subsidence suite aux apports continuels de sédiments (sable, limons) et migrations des minéraux argileux (en profondeur après dispersion, en raison des forts taux de sodium échangeable). Faute d'écoulement des eaux (chargées en sel) vers un goulet, l'accumulation et la concentration des sels n'ont cessé d'augmenter, raison pour lesquelles la mise en place d'un réseau de drainage devient impérative ; cet exutoire doit emprunter son itinéraire naturel que l'homme a dangereusement obstrué pour ses besoins d'extensions urbanistiques.

Mots clés : géochimie, Mleta, sebkha, salinité, diapirs, activité, sursaturation.

The gypseous and saline facies in the Oran area and their consequences on the resources.

\section{ABSTRACT}

More than forty thousand hectares and considerable water reserves are contaminated by salt in the study area (Mleta plain and Oran's sebkha).

Objective: The present work aims to demonstrate that the salinization in the Oran region is essentially due to saleferous formations (dating from the Trias, Lias, Éocène, Miocène and Pliocène), presents in the mountainous massifs (after the folding period of the Algerian northern Atlas). 
Methodology and results: the classic analysis, the geochemical approach and the geomorphologic study show that the evaporates come from a saline congenital substrata (primary salinization) and are later, carried downstream by rains (secondary salinization of soils and groundwater).

Conclusion and application of results : Over time, the salinity combined with erosion (both eolian and hydric due to absence of vegetation) have not only affected both soils (saline and alkali soils) and aquifers, but also contributed to the formation of a synclinal or sebkha. The latter is the result of a subsidence phenomena caused by continual sediment inputs (silts and sands) and clay minerals migration (especially at great depth due to the presence of important amounts of exchangeable sodium). That, coupled with the absence of an outlet (gully) to evacuate saline waters, have over the years, considerably contributed to the increase of salt concentrations of the waters in the region. Thus, it has become an imperative to build a drainage network in the area, in order first, to facilitate saline waters circulation and secondly, try to restore their initial itinerary, which was greatly modified because of anarchic urbanization.

Key words: geochemistry, Mleta, sebkha, salinity, diapirs, activity, oversursaturation.

\section{INTRODUCTION :}

La salinisation et l'alcalinisation des eaux et des terres est un véritable fléau en milieu semi-aride ou aride ; en Afrique, les surfaces affectées étaient estimées en 1980 à 154.277 .000 hectares dont 3.150.000 hectares en Algérie (Kovda, 1980). Pour lutter contre cet écueil majeur, il importe d'en connaitre les causes et les mécanismes ; différents facteurs sont en cause; la géologie et le climat (régime des pluies) y jouent un rôle majeur quand les autres n'en sont que les conséquences (évaporation, capillarité, ravines, oueds, cône de déjection, bourrelets, zones d'épandages). Le présent article propose d'élucider et d'examiner la manière dont se conjuguent ces facteurs, les uns aux autres; la plaine de la Mleta et la sebkha d'Oran offrent un cas d'exemple et d'étude original pour la compréhension des mécanismes de genèse d'une sebkha et la contamination (propagation du salant) des ressources (sols, eaux).

\section{Cadre général de l'étude}

Climatologie et géographie des lieux : La zone d'étude fait partie du grand bassin versant du bas Chéliff; elle est limitée au nord par le Djebel Murdjadjo (589m), au sud par les massifs du
Tessala $(1061 \mathrm{~m})$, à l'est et à l'ouest par des collines de faible altitude; le climat est de type méditerranéen, sec et chaud en été et pluvieux en hiver avec des indices de Martonne $(I=18.4)$ et de Stewart $(Q=59)$ indiquant un étage bioclimatique semi-aride et doux; le régime pluviométrique est caractérisé par des averses souvent de courte durée, très érosives.

Géologie : La constitution du bassin a débuté dès le Miocène-Pliocène; globalement les formations datant du Miocène, du Pliocène essentiellement formées de marnes bleues (Thomas, 1985; Perrodon, 1957) reposent sur un substratum secondaire autochtone schisteux. Durant le Miocène, à la faveur de la résurrection du Tell, ces formations ont été plissées et incorporées aux massifs littoraux; la sebkha et la plaine de la Mleta sont les conséquences et la résultante naturelle de cet environnement géologique, géomorphologique et hydrologique sous un climat (paléoclimat) aride, ponctués de phases de régressions et transgressions marine avec relèvement eustatique important des niveaux d'eau de la mer (Dercourt et al, 1995). La figure 1 donne un aperçu de cet ensemble. 


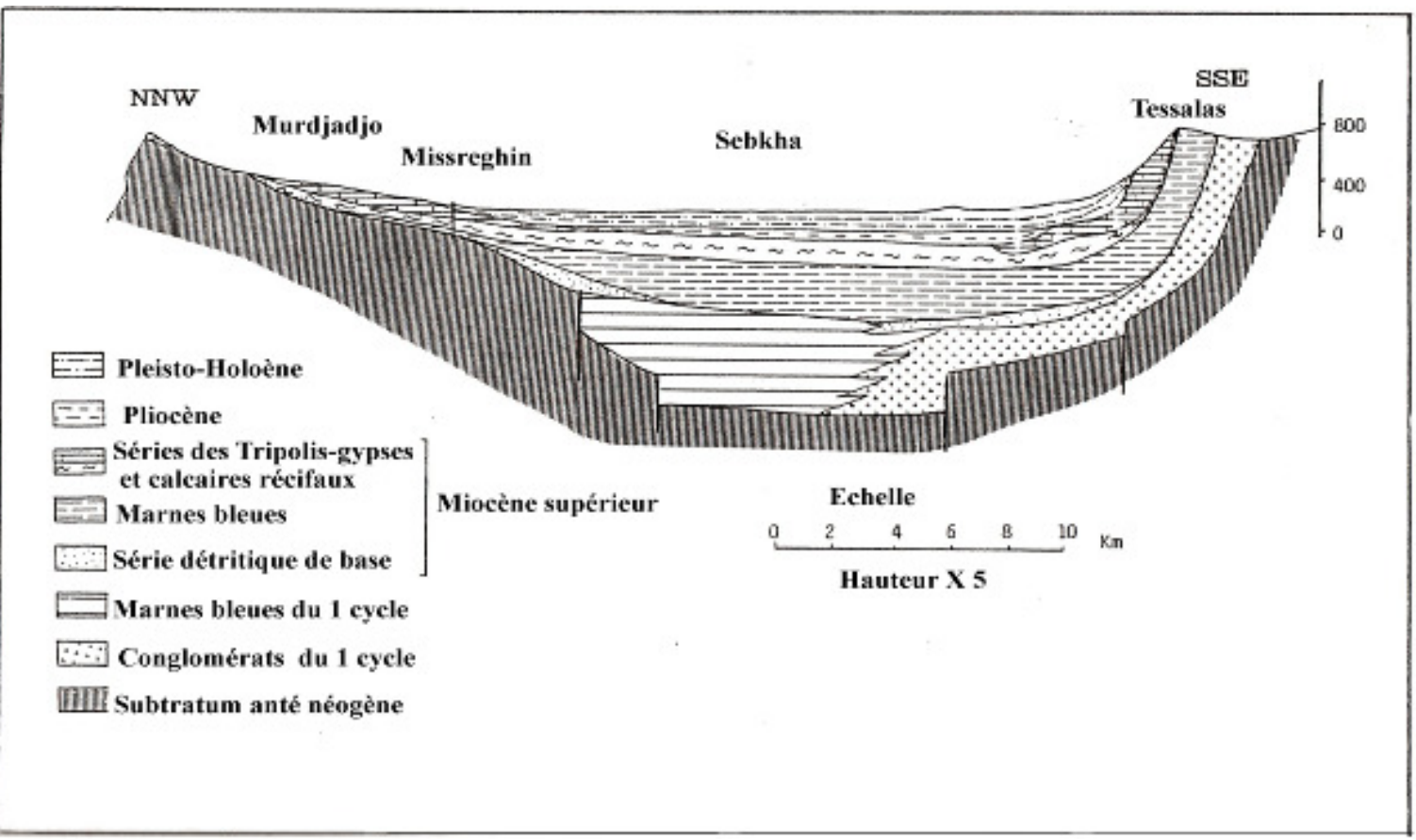

Fig.1 : Carte géologique globale (Hassani, 1987)

\section{MATERIEL ET METHODES :}

Sites d'échantillonnage et prélèvements : Les eaux prélevées et analysées proviennent de la sebkha et de la plaine de la Mleta (Bellaredj 2013); nous avons également intégré pour cette étude les eaux des oueds

(données tirées des travaux de Hassani, 1985); les sites de prélèvements sont indiqués sur la figure 2. Les numéros sur la figure renvoient aux données compilées dans les tableaux (1 et 2).

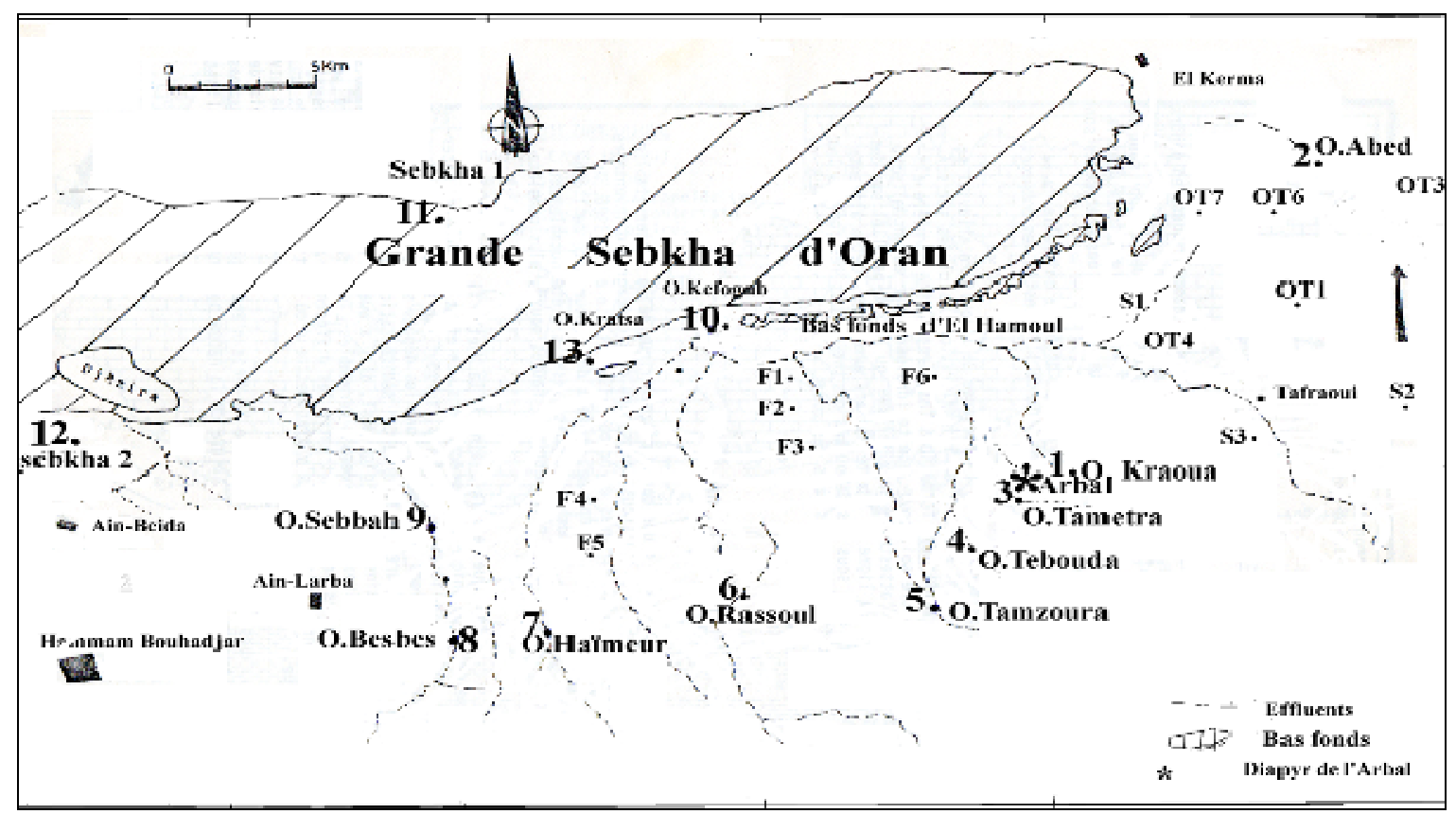

Fig. 2 : Hydrologie et sites de prélèvements 
Analyses: Les eaux (campagne octobre 2011, Bellaredj, 2013) ont été analysées au Laboratoire de l'Agence Nationales des Ressources Hydrauliques d'Oran. Les chlorures ont été dosées par argentimétrie ; les sulfates par gravimétrie, les $\mathrm{NO}_{3}$, les $\mathrm{CO}_{3}$ par colorimétrie, le sodium et le potassium par spectrophotométrie à flammes, le calcium et le magnésium par spectrophotométrie à absorption atomique.

Méthodes: Le calcul des activités des ions a été effectué au moyen de l'équation de Debye-Hückel pour

\section{RESULTATS et DISCUSSIONS :}

Origines et mécanismes de la salinisation

Caractérisation des eaux: A la lumière du Tableau $\left(n^{\circ} 1\right)$, il ressort que les eaux des oueds présentent un potentiel de salinisation très élevé. La conductivité électrique par $\mathrm{cm}$ à $25^{\circ} \mathrm{C}$ est très supérieure à 250 $\mu$ mhos/cm (Ussl, 1969 ; Valles et al, 1979; Valles et al, 1983). Le pouvoir d'alcalinisation est également très élevé, le SAR (formule de Gapon: sodium adsorption ratio $S A R=\mathrm{Na} / \sqrt{ } \mathrm{Ca}+\mathrm{Mg} / 2)$ est supérieur à 10 , à l'exception des oueds Besbes, Rassoul et Temzoura; les eaux des forages présentent les mêmes caractéristiques chimiques, fort pouvoir salinisant avec cependant un pouvoir alcalisant plus faible à l'exception des forages: F1, F2, F3 et F6, où la valeur du SAR est supérieure à 10 (USSL, 1969). Si les fortes concentrations des eaux des oueds Kraoua, Tebouda, Tametra, Tebouda, Tamzoura, Abed et des forages OT4 OT5 OT7 s'expliquent par la présence du diapyr de l'Arbal (carte géologique Arbal 1/50000' Doumergue, 1931; Perthuisot et al, 1992) la salinisation des autres oueds et forages est due essentiellement à un éventail de formations gypso-salines à l'ouest, à les forces ioniques égales ou inférieures à $0,1 \mathrm{M}$ à l'inverse des concentrations plus élevées, pour les quelles les valeurs des activités ont été obtenues grâce à l'équation de Robinson et Stokes $\left(\log \gamma=-A z^{2}\right.$ $\sqrt{ } \mathrm{l} / \mathrm{I}+\mathrm{Ba} \sqrt{\mathrm{l}}+\mathrm{bl}$; 1955), en intégrant les paramètres (a, b ) du programme Wateq (1974); les valeurs des produits de solubilités $\mathrm{K}_{\mathrm{sp}}$ ont été tirées de la bibliographie pour la Halite(log $k=1.575)$, la thénardite $(\log k=-0.331)$ et le gypse $(\log k=-4.608)$ (Naumov et al, 1971).

l'est et au nord des bassins versants du mont de Tessala alimentant en sel les oued Besbès, Haïmeur et Rassoul, et en amont sud ouest du djebel Korn (coordonnées Lambert $x=192,5 ; y=230,5$ ) et en aval du djebel Matah (coordonnées Lambert $X=201,5 ; Y=$ 240) (carte géologique d'Aïn El Berd, Institut National de Cartographie, 1977). Les eaux de l'oued Abed sont aussi très minalisées car elles proviennent de l'effluent de l'oued Kraoua (voir fig.2). Les eaux des oueds Kratz et Kefogab sont très concentrées car elles constituent l'embouchure des dépressions d'El Hamoul ; quant aux eaux de la sebkha (11 et 12), elles résultent des accumulations des sels du fait des apports par ruissellement depuis les oueds en période de fortes crues. L'approche géochimique (Tableau 2 et Fig.3) montre que la plupart des eaux des oueds et des eaux de la sebkha ou en bordure de la sebkha bien que sousaturées vis-à-vis des minéraux halite et thénardite, sont toutes sursaturées ( $\left.\log Q / K_{P S} \geq 0\right)$ vis-à-vis du gypse (à l'exception de l'oued Tamzoura). Globalement, ces états de sursaturation démontrent le caractère et l'origine gypso-saline des eaux. 


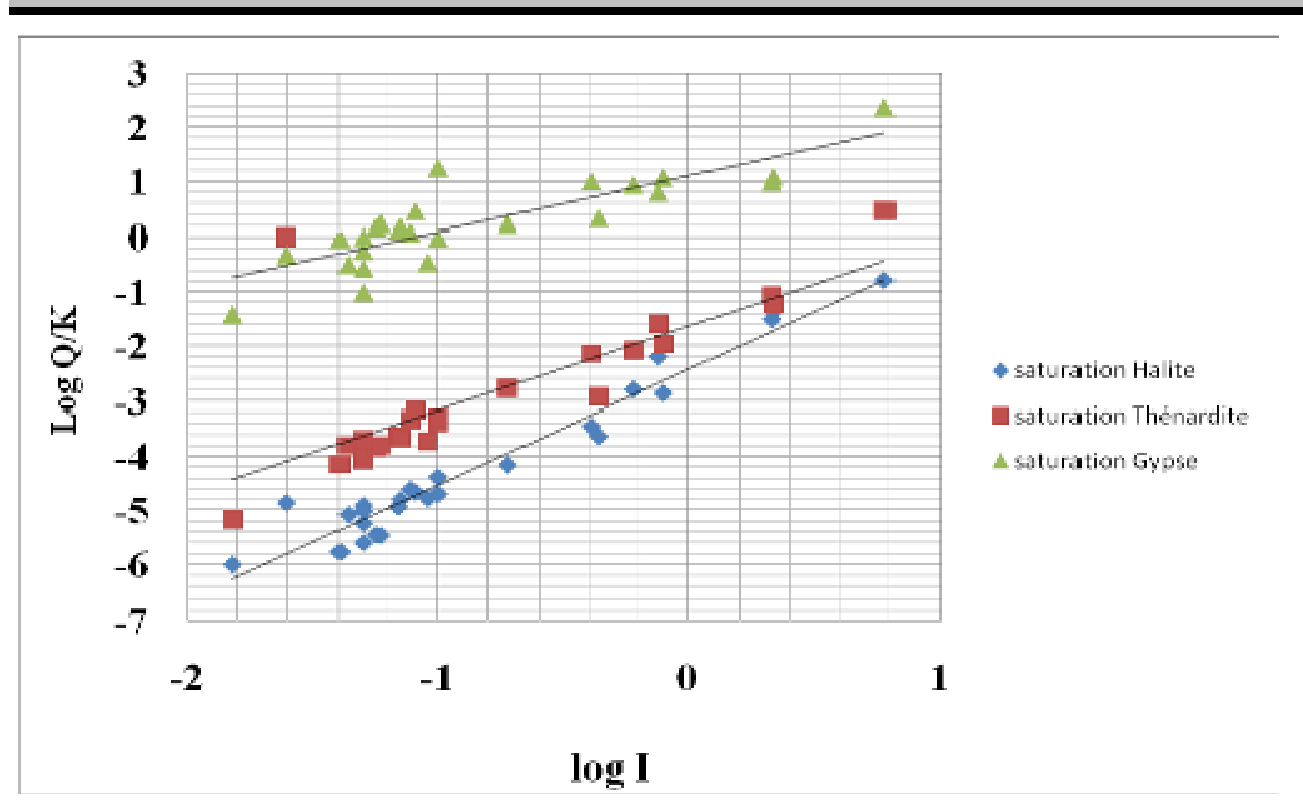

Fig.3 : Saturation des minéraux halite, thénardite et gypse en fonction de la force ionique

Paléo-environnement et source des sels : La salinisation est incontestablement d'origine marine. Elle est la résultante de phénomènes géologiques en rapport avec l'histoire de la région; en effet dès l'effondrement de la pénéplaine post hercynienne, la région d'Oran comme toute l'Algérie est inondée par la mer; au Trias, le climat très chaud (Pruvost et al, 1961) ponctué, sans aucun doute de fortes évaporations après régressions des eaux a contribué à la cristallisation très rapide des sels (sursaturation, nucléation des évaporites tels le gypse, la halite et, la thénardite,) et partant les dépôts gypso-salins. Ces mécanismes se sont produits continuellement à la faveur des séries de transgressions et de régressions des eaux, outre les modifications du niveau eustatique (Dercourt et al, 1995) et les régimes de marées qui ont favorisé l'apport de sel sur le continent; à ces dépôts triasiques, l'Oranie a connu des dépôts gypso-salins au Lias, à l'Éocène inférieur, au Miocène supérieur (Gaucher et al, 1974); de plus, à la transgression de l'Oligocène, a succédé une régression importante qui a duré plus de 5 millions d'années (Dercourt et al, 1995) favorable à des mécanismes de croissance cristallines et de litifaction, propices à la stratification des sels; par la suite, les mouvements orogéniques ont incorporés ces séries de dépôts aux massifs montagneux où par endroits le diapyrisme (à travers les failles) a fait son œuvre; les plis diapyrs et les dômes en voie de percement (ou non) constituant ainsi des sources potentielles de sel. II faut également noter que les phénomènes de subsidence ont joué une rôle non négligeable dans la formation de lagunes, ne favorisant pas les évacuations des eaux de ruissellement salées au contact des domes ou diapirs ou de sites ayant stocke des sels. II n'est pas également impossible que la surrection du tell, a contribué à la constitution de plis type auge, poches en $U$, plis plus ou moins coiffés qui aient accumulé des sels. Des dépressions ont ainsi accumulé des évaporites de la même manière, ce qui pourrait expliquer la présence de sels par endroits et non dans d'autres (Bellaredj, 2001) d'autre part, il a été démontré également la présence de formations lagunolacustres reposant sur du quaternaire datant du pliocène (à faciès astien) au sud d'Oran (Bleicher, 1874, Pallary 1901, Arambourg 1950), autre source certainement d'accumulation et de cristallisation de sel, résultat d'une autre ou plusieurs transgressions marines bien plus tard que l'effondrement du continent thyrénienn et probablement avant la résurrection total du tell. De toutes les manières, l'approche géochimique à travers les données a démontré le caractère gypsosalin des eaux.

Mécanismes de genèse de la sebkha: La sebkha d'Oran est incontestablement due à l'absence d'un passage d'évacuation des eaux de ruissèlement et de drainage. Outre les massifs littoraux (massifs de Missreghin au nord et nord ouest) qui constituent un obstacle physique, la subsidence (l'altitude de la sebkha d'Oran a diminuée de 7 mètres en un siècle seulement : de $88 \mathrm{~m}$ en 1852 à $84 \mathrm{~m}$ en 1942 à $81 \mathrm{~m}$ en 
1957, Moussa 2000), en raison des sédiments argileux et limoneux apportés par les eaux de ruissellement (sur des reliefs non boisés, et des pentes dénudées) a accentue le problème (ce phénomène n'existe pas à la Macta car les eaux peuvent emprunter des goulets sur la mer en dépit pourtant de la lagune subsidente). En fait les eaux excédentaires (de surcroit salsodiques) en saison de fortes pluies s'accumulent dans les zones d'épandage des oueds (en l'occurrence la sebkha et les bas fonds). A la décrue, ces eaux fortement chargées en Na échangeable (plus de 15\%) ont engendré la formation de solontachaks qui progressivement ont évolué en solonetz (Szalbocs, 1971; Servant, 1975; Bellaredj,1985) à cause de la dispersion et la migration des argiles en profondeur (par formation d'un horizon illuvial communément appelé en pédologie $B_{t}$, le symbole $t$ en référence au terme ton en allemand signifiant argile); l'argile en imperméabilisant les sols, a favorisé davantage le ruissellement, et donc a contribué au transport des

\section{CONSEQUENCES et PERSPECTIVES}

La plaine de la Mleta couvre environ une surface de 30000 hectares et la surface de la sebkha d'Oran a été estimée à environ 12000 ha (à partir de la carte d'état major $1 / 50000$ è $)$. Si les sols de la plaine en amont de la sebkha ne sont pas potentiellement salés, du moins en surface en raison de la pente qui favorise le lessivage et le lavage des sels grâce aux eaux de pluies, les sols de la sebkha et aux abords de celle-ci sont incontestablement salés et sodiques (Ussl, 1969 ; Boualla et al, 2012) ou salsodiques (Servant, 1975) avec des caractères morphologiques des solonetz (Szalbocs, 1971). Cependant la salinité de la plaine (en raison de la remontée capillaire des eaux salées par évaporation en été) ne constitue pas un handicap majeur à une agriculture intensive voire arboricole en veillant à une irrigation adaptée (avec apport de fraction lessivant), par contre la valorisation de la sebkha elle-même peut être envisagée (ce qui

\section{CONCLUSION ET APPLICATION DES RÉSULTATS}

La salinité (et ou la sodicité) des eaux et des sols de la sebkha et de la plaine de la Mléta est la conséquence logique d'un système qui est marqué et déterminé par deux éléments majeurs, à savoir: la présence de structures salifères congénitales et un climat désormais aride et les dépôts gypso-salins datant du trias (diapirs) ou des différentes autres époques (Lias, Éocène sédiments vers les bas fonds (il faut noter également qu'un sondage récent non loin de l'aéroport de Sénia a révélé que la sebkha et la plaine de la mléta sont constituées de plus de 70 mètres de limons, d'argiles et de sables reposant sur plus de 250 mètres de marnes du Pliocène, Moussa, 2010). Ce mécanisme se perpétuant des dizaines et des dizaines de millions d'années (dès le début de la résurrection du tell, c'està-dire dès le Miocène (Gourinard, 1958) sur des dépôts déjà congénitalement argileux (marnes bleues datant du Miocène, du Pliocène voir Fig.3), a engendré la formation d'une vaste poche gorgée en eau salée, appelée sebkha; la subsidence aidant, le phénomène s'est étendu en fonction de la configuration du terrain (pente nord ouest pour la sebkha d'Oran) et à la faveur de la texture des formations en présence : les terrains en contact à texture friable ont favorisé l'extension de la sebkha à l'inverse de ceux plus compacts, moins friables.

contribuerait également à combattre les marécages propices au développement des insectes, moustiques très prolifiques en été, autres fléaux de santé publique), si des aménagements adéquats sont réalisés avec en priorité l'évacuation des eaux par la création d'un goulet vers la mer (un drain principal). La sebkha pourrait constituer un véritable domaine pour la cotonnerie ou la céréaliculture (orge surtout) après mise en place d'un réseau de drainage. Ces deux espèces respectivement sont parfaitement adaptées à des sols dont les conductivités électriques (CE) de l'EPS (extrait de pâte saturée du sol sont inférieures à 7 ou $8 \mathrm{mmhos} / \mathrm{cm}$ à $25^{\circ} \mathrm{C}$ (Valles et al 1979); en outre le goulet éviterait une remontée des eaux salées, véritable menace qui viendraient contaminer les eaux douces de la plaine de Misshreghin ou les eaux de la région de Brédéah (à l'ouest de la sebkha).

inférieur, Miocène supérieur et Pliocène) qui le nourrissent et l'entretiennent. La gestion de ces espaces dans la zone d'étude, impose d'intégrer ces données et l'évacuation permanente des eaux de la sebkha par un réseau de drain (ou un goulet) devient impérative. 


\section{Bellaredj El Mehdi. J. Appl. Biosci. 2016 Les facies gypso-salins dans la région d'Oran et leurs conséquences sur les ressources}

Tableau 1 : Données analytiques (eaux meq/l)

\begin{tabular}{|c|c|c|c|c|c|c|c|c|c|c|c|}
\hline $\mathrm{F}=0^{*}$ & C.E mmhos/cm & Min.mg/l & $\mathrm{pH}$ & $\mathrm{Na}$ & $\mathrm{K}$ & $\mathrm{Mg}$ & $\mathrm{Ca}$ & $\mathrm{HCO}^{3}$ & $\mathrm{SO}^{4}$ & $\mathrm{Cl}$ & SAR \\
\hline F1 & 3.32 & 2520 & 7.5 & 29.8 & 0.1 & 7.23 & 3.9 & 5.9 & 10.1 & 23.4 & 12.6 \\
\hline F2 & 4.190 & 3220 & 7.4 & 32.8 & 0.1 & 14 & 4.7 & 6.5 & 12.6 & 32 & 10.75 \\
\hline F3 & 3.310 & 2460 & 7.5 & 27.6 & 0.05 & 8.4 & 3.9 & 6 & 8 & 25.1 & 11.2 \\
\hline F4 & 2.880 & 2250 & 7.8 & 24.4 & 0.1 & 6.4 & 3.7 & 6 & 9.5 & 18.8 & 4.8 \\
\hline F5 & 5.680 & 4660 & 7.4 & 39 & 0.07 & 19.7 & 7.27 & 6.7 & 22.6 & 38.4 & 2.7 \\
\hline F6 & 6.380 & 5400 & 7.3 & 50.5 & 0.1 & 17.84 & 8.9 & 6.5 & 18.6 & 51.8 & 13.9 \\
\hline OT3 & 2.220 & 1840 & 7.2 & 9.7 & 0.15 & 9.2 & 9 & 7.8 & 10.3 & 9.6 & 3.2 \\
\hline OT4 & 2.905 & 2460 & 7.2 & 14.7 & 0.2 & 9.3 & 11.8 & 5.8 & 16.4 & 14 & 4.5 \\
\hline OT5 & 3.160 & 3040 & 7.2 & 14.7 & 0.2 & 10.2 & 12.2 & 6.3 & 18.2 & 13.8 & 4.4 \\
\hline OT7 & 4.070 & 3640 & 7.1 & 26.7 & 0.2 & 11.3 & 11.4 & 7.8 & 16 & 25.7 & 7.9 \\
\hline S1 & 2.660 & 2240 & 7.2 & 12.2 & 0.2 & 10.5 & 10.8 & 11.8 & 10.7 & 11.5 & 3.7 \\
\hline S2 & 1.121 & 1040 & 7.6 & 6.4 & - & 4.5 & 2.5 & 5.1 & 1 & 6.3 & 3.4 \\
\hline S3 & 2.680 & 2240 & 7.4 & 17.7 & 0.2 & 7.7 & 6.9 & 4.6 & 9.4 & 18.6 & 6.5 \\
\hline Kraoua & 210.6 & 138572 & 8.4 & 2050 & 6.28 & 39.97 & 55.04 & 2.67 & 120.9 & 1849.3 & 297.5 \\
\hline Abed & 35.4 & 21948 & 7.9 & 410 & 8.5 & 157.6 & 40 & 3.36 & 193.7 & 356.4 & 41.3 \\
\hline Tametra & 17.5 & 10850 & 8.4 & 144 & 0.6 & 4.9 & 18 & 0.5 & 31.9 & 127.0 & 42.6 \\
\hline Tebouda & 32.6 & 20212 & 8.4 & 420 & 0.9 & 25.5 & 40 & 2.25 & 137.4 & 373.5 & 73.4 \\
\hline Tamzour & 3 & 1862 & 8.3 & 15.8 & 0.2 & 8.9 & 6.6 & 4.1 & 20.2 & 9.2 & 2 \\
\hline Rassoul & 5.2 & 3224 & 8.4 & 38.5 & 0.4 & 14.3 & 10 & 3.5 & 28.5 & 26.7 & 5.6 \\
\hline Haîmeur & 11.8 & 7316 & 8 & 84 & 0.6 & 22.8 & 32.9 & 2.7 & 68.9 & 62.2 & 15.9 \\
\hline Besbes & 57.4 & 3534 & 8.3 & 27.5 & 0.4 & 12.9 & 15 & 2.7 & 15 & 34.9 & 7.4 \\
\hline Sebbah & 6.1 & 3782 & 8.4 & 40 & 0.4 & 16.4 & 13 & 2.1 & 33.5 & 32.9 & 10.5 \\
\hline Kefogab & 23.4 & 14508 & 8.5 & 198 & 0.5 & 78.6 & 24 & 2.4 & 121.9 & 156.7 & 59.5 \\
\hline Sebkha1 & 127.7 & 79174 & 7.8 & 1640 & 9 & 245.9 & 115 & 1.5 & 204.9 & 1523.6 & 122.4 \\
\hline Sebkha2 & 69.8 & 43276 & 8.9 & 775 & 1.7 & 141.5 & 20 & 5.9 & 162.4 & 690.5 & 86.4 \\
\hline Sebkha1 & 127.7 & 79174 & 7.8 & 1640 & 9 & 245.9 & 115 & 1.5 & 204.9 & 1523.6 & 122.4 \\
\hline O.kratsa & 155.7 & 95534 & 8.1 & 3700 & 3.51 & 576.9 & 31 & 8.2 & 3198 & 1946.2 & 212 \\
\hline
\end{tabular}

$\mathrm{F}-\mathrm{O}^{*}=$ forages et oueds

Tableau 2 : Activités des ions, forces ioniques et états de saturation

\begin{tabular}{|c|c|c|c|c|c|c|c|c|c|c|c|}
\hline \multirow{2}{*}{ Ech } & \multirow{2}{*}{$\mathrm{Fl}^{*}$} & \multicolumn{9}{|c|}{ Activités } & \multicolumn{4}{|c|}{ LogQ//Ks } & \multirow{2}{*}{ Log FI } \\
\cline { 3 - 13 } & & $\mathrm{Ca}$ & $\mathrm{Mg}$ & $\mathrm{Na}$ & $\mathrm{SO}_{4}$ & $\mathrm{Cl}$ & $\mathrm{HCO}_{3}$ & Hal. $^{+}$ & Thern+ & Gypse+ & \\
\hline F1 & 0.025 & 0.556 & 0.59 & 0.857 & 0.539 & 0.851 & 0.858 & -4.86 & --3.59 & -0.319 & -1.602 \\
\hline F2 & 0.09 & 0.407 & 0.450 & 0.773 & 0.358 & 0.758 & 0.773 & -4.78 & -3.72 & -0.456 & -1.04 \\
\hline F3 & 0.049 & 0.483 & 0.517 & 0.817 & 0.445 & 0.807 & 0.819 & -4.91 & -3.85 & -0.565 & -1.30 \\
\hline F4 & 0.043 & 0.483 & 0.531 & 0.825 & 0.464 & 0.816 & 0.827 & -5.08 & -3.80 & -0.495 & -1.36 \\
\hline F5 & 0.076 & 0.431 & 0.471 & 0.787 & 0.385 & 0.774 & 0.791 & -4.61 & -3.34 & $\underline{\mathbf{0 . 0 8}}$ & -1.11 \\
\hline F6 & 0.099 & 0.400 & 0.444 & 0.769 & 0.350 & 0.753 & 0.773 & -4.39 & -3.38 & -0.02 & -1 \\
\hline S1 & 0.049 & 0.483 & 0.517 & 0.817 & 0.445 & 0.807 & 0.819 & -5.01 & -4.08 & 0.0031 & -1.3 \\
\hline S2 & 0.015 & 0.628 & 0.646 & 0.883 & 0.608 & 0.879 & 0.884 & -6.00 & -5.18 & -1.41 & -1.82 \\
\hline S3 & 0.05 & 0.481 & 0.515 & 0.815 & 0.443 & 0.805 & 0.818 & -5.24 & -3.97 & -0.251 & -1.3 \\
\hline OT3 & 0.04 & 0.508 & 0.539 & 0.829 & 0.474 & 0.821 & 0.831 & -5.77 & -4.15 & -0.043 & -1.39 \\
\hline OT4 & 0.055 & 0.469 & 0.505 & 0.809 & 0.429 & 0.799 & 0.812 & -5.45 & -3.83 & $\underline{\mathbf{0 . 1 7 5}}$ & -1.25 \\
\hline OT5 & 0.058 & 0.463 & 0.499 & 0.806 & 0.422 & 0.795 & 0.808 & -5.46 & -3.8 & $\underline{\mathbf{0 . 2 4 5}}$ & -1.23 \\
\hline
\end{tabular}




\section{Bellaredj El Mehdi. J. Appl. Biosci. 2016 Les facies gypso-salins dans la région d'Oran et leurs}

conséquences sur les ressources

\begin{tabular}{|c|c|c|c|c|c|c|c|c|c|c|c|}
\hline OT7 & 0.068 & 0.444 & 0.482 & 0.795 & 0.400 & 0.783 & 0.798 & -4.94 & -3.63 & $\underline{\mathbf{0 . 1 1 8}}$ & -1.16 \\
\hline O.Kraoua & 2.17 & 0.386 & 0.654 & 0.806 & 0.115 & 0.531 & 0.563 & -1.36 & -1.21 & $\underline{\mathbf{1 . 0 8}}$ & 0.336 \\
\hline O.Abed & 0.78 & 0.246 & 0.288 & 0.707 & 0.197 & 0.613 & 0.667 & -2.87 & -1.96 & $\underline{\mathbf{1 . 0 7}}$ & -0.1 \\
\hline O.Tamina & 0.43 & 0.338 & 0.322 & 0.660 & 0.274 & 0.700. & 0.668 & -3.64 & -2.92 & $\underline{\mathbf{0 . 3 3}}$ & -0.36 \\
\hline O.Tebouda & 0.6 & 0.239 & 0.301 & 0.636 & 0.165 & 0.595 & 0.645 & -2.8 & -2.08 & $\underline{\mathbf{0 . 9 4 3}}$ & -0.22 \\
\hline O Temzou & 0.05 & 0.418 & 0.515 & 0.815 & 0.443 & 0.805 & 0.818 & -5.6 & -3.7 & -001 & -1.3 \\
\hline OGhassoul & 0.08 & 0415 & 0.457 & 0.778 & 0.367 & 0.764 & 0.781 & -4.7 & -3.3 & $\underline{\mathbf{1 . 2 5}}$ & -1 \\
\hline OHaimer & 0.19 & 0.195 & 0.380 & 0.718 & 0.260 & 0.694 & 0.723 & -4.16 & -2.77 & $\underline{\mathbf{0 . 2 4}}$ & -0.72 \\
\hline OBesbes & 0.07 & 0.431 & 0.471 & 0.787 & 0.385 & 0.774 & 0.771 & -4.8 & -3.67 & $\underline{\mathbf{0 . 1 7 7}}$ & -1.15 \\
\hline OSebbah & 0.08 & 0.420 & 0.462 & 0.781 & 0.373 & 0.767 & 0.785 & -4.67 & -3.17 & $\mathbf{0 . 4 5 5}$ & -1.09 \\
\hline Kefrogab & 0.4 & 0.263 & 0.324 & 0.663 & 0.324 & 0.629 & 0.671 & -3.46 & -2.13 & $\underline{\mathbf{1 . 0 0}}$ & -0.39 \\
\hline Sebha1 & 2.15 & 0.299 & 0.406 & 0.804 & 0.184 & 0.566 & 0.619 & -1.51 & -1.06 & $\underline{\mathbf{0 . 9 9}}$ & 0.33 \\
\hline Sebkha2 & 0.75 & 0.247 & 0.288 & 0.706 & 0.197 & 0.614 & 0.667 & -2.20 & -1.58 & $\underline{\mathbf{0 . 8 0}}$ & -0.12 \\
\hline Kratz & 6.1 & 1.02 & 1.978 & .1 .446 & 0.057 & 0.579 & 0.582 & -0.79 & $\underline{\mathbf{0 . 4 8}}$ & $\underline{\mathbf{2 . 3 6}}$ & 0.78 \\
\hline
\end{tabular}

${ }^{*} \mathrm{Fl}$ : force ionique + Sursaturation vis-à-vis des minéraux halite, thénardite et gypse.

\section{BIBLIOGRAPHIE :}

Arambourg C, 1950. Contribution à l'étude des formations laguno-lacustres des environs d'Oran. Bull. Soc. Hist. Nat. Afr. Nord $4: 20-$ 29.

Bellaredj AE, 2013. Caractérisation des eaux souterraines de la plaine de la Mleta par application des méthodes statistiques multivariées et de la modélisation géochimique. Thèse Magister. Université d'Oran, Algérie.

Bellaredj B, 2001. Paleoenvironnement - formations géologiques et propagation du salant (salinisation primaire). XX1 Séminaire National des Sciences de la terre. Université de Tlemcen, Algérie.

Bellaredj B, 2000. Méthodologie d'approche des problèmes de salinisation et d'alcalinisation des sols. Avant-projet de développement intégré et lutte contre la remontée des eaux à Oued Souf. Congrès CRSTRA. Oued Souf,. Algérie. Octobre 2000.

Bellaredj B, 1985. Analyse des causes de baisse de productivité du clémentinier sur la plaine de la Habra. Thèse Docteur - Ingénieur INP Toulouse, France.

Bleicher M, 1874. Recherches sur l'origine des éléments lithologiques des terrains tertiaires et quaternaires des environs d'Oran. Comptes Rendus. Acad. Sci 78 : 700-701.

Carte Géologique, Aïn El Berd. 1977. Institut National de Cartographie. Alger.

Boualla N, Benziane A et Derriche Z. 2012. Origne de la salinisation des terres de la plaine de la
Mleta (bordure sud bassin Sebkha Oran). Journal of Applied BioSciences 53: 37873796.

Dercourt J.and Paquet J. 1995. Géologie. Objets et méthodes. Dunod ed, Paris. 404pp.

Doumergue F, 1931. Carte Géologique Arbal $1 / 50000$ è. Service Géologique de l'Algérie.

Gaucher G and Burdin S. 1974, Géologie, géomorphologie et hydrologie des terres salées. PUF ed. Paris. $234 \mathrm{pp}$.

Gourinard Y, 1958. Recherches sur la géologie du littoral oranais. Publ. Serv. Carte. Géol. Algérie, Nouv. Sér, 6. Alger. 111pp.

Hassani M.I, 1987. Hydrogéologie d'un bassin endoréique - Le bassin versant de la grande sebkha d'Oran. Thèse de Doctorat. Université Scientifique Technologique et Médicale de Grenoble.

Ivanova EN and Rozanov AN, 1939. Classification of salinized soils. Pedology 7: 44-52.

Kovda VA. 1980. Problem of combating salinization of irrigated soils. Centre for International Projects ed. Moscow. 274 pp.

Moussa K, 2007. Étude d'une sebkha: la sebkha d'Oran. Thèse d'État. Université d'Oran. Algérie.

Naumov CB, Ryzhenko BN and Khodakovsky TL. 1971. Thermodynamics data atlas. Atomitzdat Ed. Moscou.239pp.

Pallary $P, 1901$. Sur mollusques fossiles terrestres fluviatiles et saumâtres de l'Algérie. Mem. Soc. Géol. Fr. 22. 
Perrodon, A, 1957. Étude géologique des bassins sédimentaires. Sci. Terre, 16, 2, 195-227.

Perthuisot, V., Rouvier, H. 1992. Les diapyrs du maghreb central et oriental: des appareils variés, résultat d'une évolution structurale et petrogénétique complexe. Bull. Soc. Geol. France, 163, 6, 751-760.

Pruvost, P, Roch, E et Tadashi, S. 1961. Le concept de cycle sédimentaire: retouches et compléments. Comptes Rendus. Acad. Sci, $253: 1742$ - 1744.

Robinson R. and Stokes RH, 1955. Electrolyte solution. Butterworths Scientific Publications ed. London. 559pp.

Servant J, 1975. Contribution à l'étude pédologique des terrains halomorphes. Thèse d'État. USTL. Montpellier.
Szalbocks I, 1971. European solonetz soils and their reclamation. Akademial Budapest.203p.

Thomas G. 1985. Géodynamique d'un basin intramontagneux. Le bassin du Bas Chélif occidental(Algérie) durant le Mio-PlioQuaternaire. Thèse d'État, Université de Pau.

Ussl, 1969. Diagnosis and improvement of saline and alkali soils. Agriculture Handbook 60, L.A.Rchards ed, Riverside (California). 160 pp.

Valles V, Valles AM et Dosso M. 1983. Irrigation des sols salés et doses de lessivage. Cah. ORSTOM. Série Pédologie.XX, 2, 119-127.

Valles V. et Dosso M, 1979. Problèmes posés par I'utilisation des eaux salées pour l'irrigation. Rapport Groupements d'études et de réalisations des Sociétés d'Aménagement Régional. GERSAR. Ed, Paris. 45pp. 